\title{
ON SOME CHARACTERIZATIONS OF ANTIPODAL PARTIAL CUBES
}

\author{
Norbert POLAT \\ I.A.E., Université Jean Moulin (Lyon 3) \\ 6 cours Albert Thomas \\ 69355 Lyon Cedex 08, France
}

e-mail: norbert.polat@univ-lyon3.fr

\begin{abstract}
We prove that any harmonic partial cube is antipodal, which was conjectured by Fukuda and K. Handa, Antipodal graphs and oriented matroids, Discrete Math. 111 (1993) 245-256. Then we prove that a partial cube $G$ is antipodal if and only if the subgraphs induced by $W_{a b}$ and $W_{b a}$ are isomorphic for every edge $a b$ of $G$. This gives a positive answer to a question of Klavžar and Kovše, On even and harmonic-even partial cubes, Ars Combin. 93 (2009) 77-86. Finally we prove that the distance-balanced partial cube that are antipodal are those whose pre-hull number is at most 1 .
\end{abstract}

Keywords: diametrical graph, harmonic graph, antipodal graph, distancebalanced graph, partial cube, pre-hull number.

2010 Mathematics Subject Classification: 05C12, 05C75.

\section{REFERENCES}

[1] H.-J. Bandelt and A.W.M. Dress, A canonical decomposition theory for metrics on a finite set, Adv. Math. 92 (1992) 47-105.

doi:10.1016/0001-8708(92)90061-O

[2] V. Chepoi, Isometric subgraphs of Hamming graphs and d-convexity, Cybernetics 24 (1988) 6-11. doi:10.1007/BF01069520

[3] V. Chepoi, K. Knauer and T. Marc, Partial cubes without $Q_{3}^{-}$minors, CoRR abs/1606.02154 (2016) (submitted).

[4] J. Desharnais, Maille et Plongements de Graphes Antipodaux, Mémoire de Maitrise (Université de Montréal, 1993). 
[5] D. Djoković, Distance preserving subgraphs of hypercubes, J. Combin. Theory Ser. B 14 (1973) 263-267. doi:10.1016/0095-8956(73)90010-5

[6] D. Eppstein, The lattice dimension of a graph, European J. Combin. 26 (2005) 585592. doi:10.1016/j.ejc.2004.05.001

[7] M. Faghani, E. Pourhadi and H. Kharazi, On the new extension of distance-balanced graphs, Trans. Comb. 5 (2016) 23-34.

[8] V.V. Firsov, Isometric embedding of a graph into a Boolean cube, Cybernetics 1 (1965) 112-113. doi:10.1007/BF01074705

[9] K. Fukuda and K. Handa, Antipodal graphs and oriented matroids, Discrete Math. 111 (1993) 245-256. doi:10.1016/0012-365X(93)90159-Q

[10] F. Glivjak, A. Kotzig and J. Plesník, Remarks on the graphs with a central symmetry, Monatsh. Math. 74 (1970) 302-307. doi:10.1007/BF01302697

[11] F. Göbel and H.J. Veldman, Even graphs, J. Graph Theory 10 (1986) 225-239. doi:10.1002/jgt.3190100212

[12] R. Hammack, W. Imrich and S. Klavžar, Handbook of Product Graphs, Second Edition (CRC Press, 2011).

[13] K. Handa, Bipartite graphs with balanced (a,b)-partitions, Ars Combin. 51 (1999) 113-119.

[14] A. Ilíc, S. Klavžar and M. Milanovíc, On distance-balanced graphs, European J. Combin. 31 (2010) 732-737. doi:10.1016/j.ejc.2009.10.006

[15] J. Jerebic, S. Klavžar and D.F. Rall, Distance-balanced graphs, Ann. Comb. 12 (2008) 71-79. doi:10.1007/s00026-008-0337-2

[16] S. Klavžar and M. Kovše, On even and harmonic-even partial cubes, Ars Combin. 93 (2009) 77-86.

[17] S. Klavžar and H.M. Mulder, Partial cubes and crossing graphs, SIAM J. Discrete Math. 15 (2002)) 235-251. doi:10.1137/S0895480101383202

[18] A. Kotzig, Centrally symmetric graphs, Czechoslovak Math. J. 93 (1968) 605-615.

[19] A. Kotzig and P. Laufer, Generalized S-Graphs, preprint CRM-779, Centre de recherches mathématiques (Université de Montréal, 1978).

[20] T. Marc, There are no finite partial cubes of girth more than 6 and minimum degree at least 3, European J. Combin. 55 (2016) 62-72. doi:10.1016/j.ejc.2016.01.005 
[21] H.M. Mulder, The structure of median graphs, Discrete Math. 24 (1978) 197-204. doi:10.1016/0012-365X(78)90199-1

[22] H.M. Mulder, n-cubes and median graphs, J. Graph Theory 4 (1980) 107-110. doi:10.1002/jgt.3190040112

[23] S. Ovchinnikov, Partial cubes: structures, characterizations, and constructions, Discrete Math. 308 (2008) 5597-5621. doi:10.1016/j.disc.2007.10.025

[24] K.R. Parthasarathy and R. Nandakumar, Unique eccentric point graphs, Discrete Math. 46 (1983) 69-74.

doi:10.1016/0012-365X(83)90271-6

[25] N. Polat, Netlike partial cubes I. General properties, Discrete Math. 307 (2007) $2704-2722$. doi:10.1016/j.disc.2007.01.018

[26] N. Polat and G. Sabidussi, On the geodesic pre-hull number of a graph, European J. Combin. 30 (2009) 1205-1220. doi:10.1016/j.ejc.2008.09.022

[27] G. Sabidussi, Graphs without dead ends, European J. Combin. 17 (1996) 69-87. doi:10.1006/eujc.1996.0006

[28] P. Winkler, Isometric embeddings in products of complete graphs, Discrete Appl. Math. 7 (1984) 221-225.

doi:10.1016/0166-218X(84)90069-6

Received 28 April 2017 Revised 13 September 2017 Accepted 13 September 2017 